\title{
Do signal transduction cascades influence survival in triple-negative breast cancer? A preliminary study
}

This article was published in the following Dove Press journal:

OncoTargets and Therapy

26 May 2016

Number of times this article has been viewed

\author{
Jan-Niclas Mumm \\ Alexandra C Kölbl \\ Udo Jeschke \\ Ulrich Andergassen \\ Department of Obstetrics and \\ Gynaecology, Ludwig-Maximilians \\ University of Munich, Munich, \\ Germany
}

Correspondence: Udo Jeschke Department of Obstetrics and Gynaecology, Ludwig-Maximilians University of Munich, Maistrasse II, 80337 Munich, Germany

Tel +49894400 54III

Fax +49894400 547I5

Email udo.jeschke@med.uni-muenchen.de
Background: Triple-negative breast cancer (TNBC) is a rather aggressive form of breast cancer, comprised by early metastasis formation and reduced overall survival of the affected patients. Steroid hormone receptors and the human epidermal growth factor receptor 2 are not overexpressed, limiting therapeutic options. Therefore, new treatment options have to be investigated. The aim of our preliminary study was to detect coherences between some molecules of intracellular signal transduction pathways and survival of patients with TNBC, in order to obtain some hints for new therapeutical solutions.

Methods: Thirty-one paraffin-embedded tumor tissue samples, which were determined to be negative for steroid hormone receptors as well as human epidermal growth factor receptor 2 , were immunohistochemically stained for a number of signal transduction molecules from several signaling pathways. $\beta$-Catenin, HIF1 $\alpha$, MCL, Notch1, LRP6, XBP1, and FOXP3 were stained with specific antibodies, and their staining was correlated with patient survival by Kaplan-Meier analyses.

Results: Only two of the investigated molecules have shown correlation with overall survival. Cytoplasmic staining of HIF1 $\alpha$ and centro-tumoral lymphocyte FOXP3 staining showed statistically significant correlations with survival.

Conclusion: The coherence of signal transduction molecules with survival of patients with TNBC is still controversially discussed in the literature. Our study comprises one more mosaic stone in the elucidation of these intracellular processes and their influences on patient outcome. Lots of research still has to be done in this field, but it would be worthwhile as it may offer new therapeutic targets for a group of patients with breast cancer, which is still hard to treat.

Keywords: signal transduction cascades, immunohistochemistry, Kaplan-Meier analysis

\section{Introduction}

Breast cancer is still a prevalent malignant disease worldwide, and the most frequent cause of death in women. ${ }^{1}$ Although lethality has decreased over the last 40 years, still $30 \%$ of the affected patients die from the consequences of breast cancer. ${ }^{2}$

"Triple-negative breast cancer" (TNBC) is pathologically defined by a low or almost absent expression of estrogen receptor, progesterone receptor, and human epidermal growth factor receptor 2 (Her2). ${ }^{3,4}$ This aggressive form of breast cancer often affects rather young women and is accompanied by a frequent development of visceral metastases, a high risk of recurrence, and a reduced overall survival (OAS) independent of tumor size, staging, and lymph node affection. ${ }^{5,6}$ The low expression of hormone receptor and Her2 receptor also restricts the possibilities of treatment options in a manner that TNBCs are treated postoperatively with a dose-dense or 
metronome chemotherapy using platin or anthracycline and taxan containing chemotherapy and radiation. ${ }^{7} \mathrm{New}$ therapeutic strategies using poly-ADP-ribose-polymerase or vascular endothelial growth factor inhibitors, in fact, increase survival time but have strong side effects. ${ }^{8-11}$

Therefore, there is a further need of new therapeutical strategies, targeting inter- and intracellular signal transduction pathways, regulating cell adhesion and proliferation. One of those signal transduction cascades could be the Notch1 pathway, which plays a role in normal breast development and cell fate determination and is, especially in TNBC, activated in an aberrant manner. An inhibition of this pathway had been shown to result in an antitumor activity by cell cycle arrest, apoptosis, and disruption of angiogenesis. ${ }^{12,13}$ HIF $1 \alpha$ is also a member of the Notchsignaling cascade, associated with a poor prognosis for the affected patients by promoting carcinoma onset and formation of lung metastasis. A reduction of expression resulted in reduced primary tumor growth, suppression of lung metastasis, and prolonged survival. ${ }^{14}$ Together with $\mathrm{XBP} 1, \mathrm{HIF} 1 \alpha$ forms a transcriptional complex that is responsible for onset of tumorigenicity. An inhibition of XBP1 results in reduced tumor growth, giving hints that it could be used as a therapeutic target. ${ }^{15}$ Another signal transduction pathway, which might be of therapeutical interest, is the Wnt/ $\beta$-catenin pathway, as it regulates cell cycle, cell growth, and tumor progression, and seems to be responsible for poor clinical outcomes. An inhibition of Wnt receptor is already known for its therapeutical use, as it induces LRP6 degradation. ${ }^{16}$ LRP6 does not seem to have a correlation with estrogen receptor, progesterone receptor, and Her2, but its use as a drug target receptor significantly prolonged survival time in a mouse model. ${ }^{17} \mathrm{MCL} 1$, another signal molecule within the $\mathrm{Wnt} / \beta$-catenin pathway, modulates mitochondrial physiology and is associated with enhanced metastasis formation and decreased disease-free survival (DFS) and could also be of therapeutic interest. ${ }^{18} \mathrm{~A}$ transcription factor, which is highly expressed in tumor cells, is FOXP3, a potent repressor of several oncogenes, which could be a target for new therapeutical interventions of TNBC. It is involved in TNBC susceptibility and prognosis, but the prognosis is dependent on the cellular localization of FOXP3. ${ }^{19}$ If it is found in the cytoplasm, it is a marker for poor OAS, but if it is found in the nucleus, OAS is markedly improved. ${ }^{20}$

We show here a correlation of these signal transduction molecules with survival of patients with TNBC in a retrospective analysis of survival data combined with immunohistochemical staining of tissue samples.

\section{Materials and methods Patient samples}

Tissue samples of breast cancer patients, who were treated between 2001 and 2002 at the Department of Obstetrics and Gynaecology of Ludwig Maximilians University of Munich, were collected and embedded in paraffin in the Department of Pathology of Ludwig Maximilians University of Munich (ethical votes compliant to the Declaration of Helsinki: LMU 048-08 and 148-12) and written informed consent was obtained. Hormone receptor status and Her2 status were determined pathologically. Thirty-one samples were regarded as TNBC. The patients who donated these samples had an average age of 62 years. Tumor samples were further classified as shown in Table 1.

Table I Patient/tumor characteristics of the tissue samples used for immunohistochemical staining

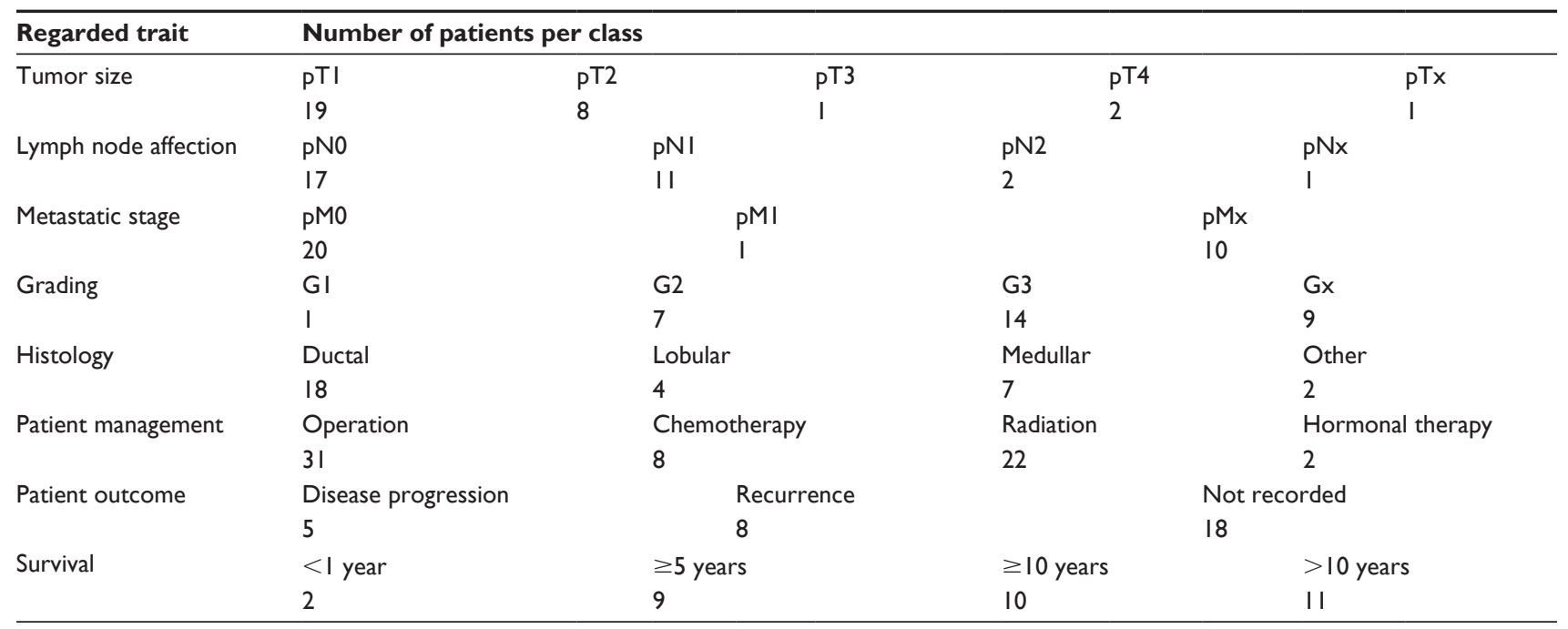




\section{Immunohistochemical staining}

The paraffin-embedded tissue samples were cut by sliding microtome and transferred onto specially covered microscope slides (SuperFrost Plus; Menzel GmbH, Berlin, Germany). The slides were air-dried overnight at $56^{\circ} \mathrm{C}-58^{\circ} \mathrm{C}$. For immunohistochemistry, paraffin was removed by a 20 -minute incubation of the slides in Xylol (Merck, Darmstadt, Germany) and subsequent washes in different dilutions of ethanol $(100 \%, 90 \%$, $75 \%$ ). Thereafter, slides were incubated in $3 \% \mathrm{H}_{2} \mathrm{O}_{2}$ in methanol (VWR International, Radnor, PA, USA) to prevent unspecific staining of tissue samples. To reconstitute the antigens, slides were washed first in ethanol $(100 \%, 70 \%, 50 \%)$ and afterward in water, and boiled in $0.1 \mathrm{M} \mathrm{Na}$ citrate (Merck) buffer (pH 6) for 5 minutes. The slides were cooled down and then washed in water and twice in phosphate-buffered saline (Biochrom, Cambridge, UK). To prevent unspecific binding of the primary antibody, samples are blocked in 10\% normal goat serum (Vector Laboratories, Burlingame, CA, USA) for 20 minutes, blocking solution was removed, and primary antibodies were applied in the appropriate concentrations (Table 2).

Incubation of primary antibodies was carried out at $4^{\circ} \mathrm{C}$ for 18 hours. Slides were washed with phosphate-buffered saline and incubated with the biotinylated secondary antibody, which is included in the staining kit (Vector Laboratories), for 30 minutes at room temperature. Thereafter, the samples were treated with avidin and biotinylated horseradish peroxidase $(\mathrm{ABC})$ reagent (Vector Laboratories) for 30 minutes, and then DAB reagent (DAKO, Glostrup, Denmark) diluted in $\mathrm{H}_{2} \mathrm{O}_{2}$ was added to the slides for 1 minute. Enzyme reaction was stopped by washing the slides in water. Nuclei were then counterstained by Hemalaun (Applichem, Darmstadt, Germany) for 5 minutes before slides were again dehydrated by ethanol $(70 \%, 96 \%, 100 \%)$ and xylol and embedded in Eukitt (Medite, Burgdorf, Germany).

To be sure of antibody function and to determine optimal dilutions, positive and isotype controls were carried out.

\section{Microscopy}

Staining of the samples was observed and evaluated by two independent persons by a Leitz Diaplan light microscope
(Ernst Leitz GmbH, Wetzlar, Germany) (Figure 1). Evaluation was carried out following the Immune Reactive Score (IRS), calculated from staining intensity and number of stained cells. ${ }^{21}$ The IRS is then set into reference to survival data.

\section{Statistical evaluation}

Statistical analysis was performed by SPSS (SPSS Inc., Chicago, IL, USA) version 22.0. Survival curves were generated by Kaplan-Meier analysis. Cutoff values for statistical analysis were set in reference to the median of IRS staining, thereby dividing the patient samples in similar groups.

\section{Results}

Different molecules, which play a role in signal transduction cascades, were stained immunohistochemically in tissue sections of patients with TNBC. Via Kaplan-Meier analysis, correlations between staining and patient survival were investigated. A statistically significant correlation could be found for only two signaling molecules: HIF $1 \alpha$ staining of the cytoplasm (C) and FOXP3 staining of lymphocytes in the center of the tumor tissue (CT; $P=0.004$ and $P=0.015$, respectively). For a nuclear (N) HIF1 $\alpha$ staining and FOXP3 staining of lymphocyte clusters in peritumoral regions (TLS) and of the tumor itself (Tum), no correlations were found ( $P=0.934, P=0.230$, and $P=0.433$ ). All further investigated molecules, which contribute to intracellular signal transduction, did not show any statistically significant coherences with survival (Figure 2 and Table 3 ).

\section{Discussion and conclusion}

The results presented in our rather preliminary study are in part compliant with former research results, but there are some contrasts as well. HIF $1 \alpha$ staining was already found to have a correlation with survival and was furthermore regarded as a poor prognostic factor for survival of breast cancer patients. ${ }^{22,23}$ With the immunohistochemical staining, we could reassure this finding, especially when cytoplasmic HIF $1 \alpha$ is regarded. That means, the coherence between HIF $1 \alpha$ and survival is dependent on the intracellular

Table 2 Primary antibodies used for staining

\begin{tabular}{|c|c|c|c|}
\hline Antibody & Clonality & Working dilution & Distributor \\
\hline Anti-HIFI $\alpha$ & Monoclonal rabbit-lgG & $\mathrm{I}: 2,000$ & Sigma Aldrich \\
\hline Anti- $\beta$-catenin & Polyclonal rabbit-lgG & $\mathrm{I}: 300$ & Diagnostic Biosystems \\
\hline Anti-XBPI & Monoclonal rabbit-lgG & $\mathrm{I}: 400$ & Sigma Aldrich \\
\hline Anti-FOXP3 & Monoclonal mouse lgGI & $\mathrm{I}: 300$ & Abcam \\
\hline Anti-Notch I & Monoclonal mouse-lgG & $\mathrm{I}: 100$ & Sigma Aldrich \\
\hline Anti-MCLI & Monoclonal rabbit-lgG & $\mathrm{I}: \mathrm{I}, 000$ & Abcam \\
\hline Anti-LRP6 & Rabbit-lgG & $\mathrm{I}: 80$ & Millipore \\
\hline
\end{tabular}




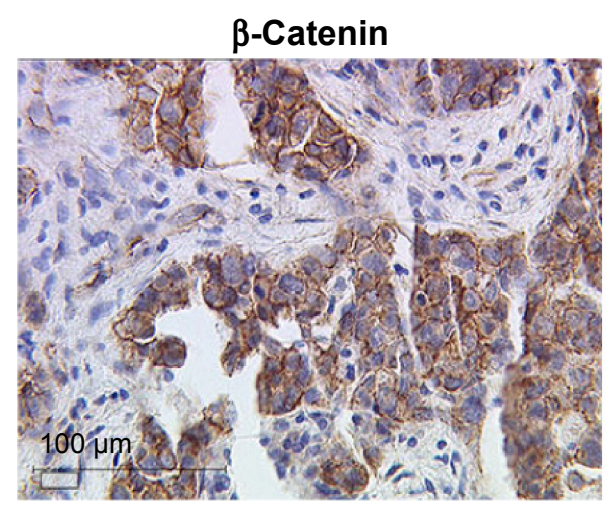

HIF1 $\alpha$

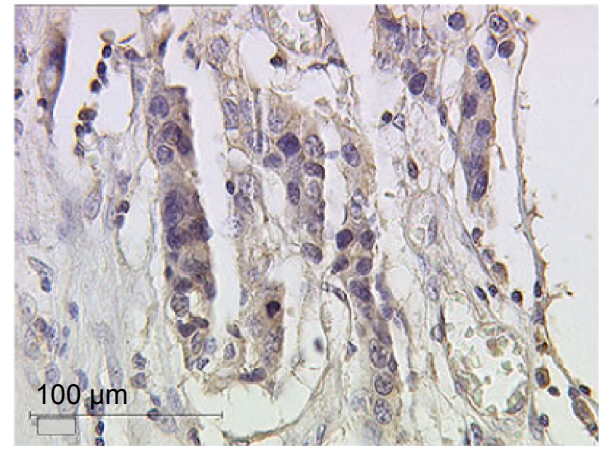

\section{Notch1}

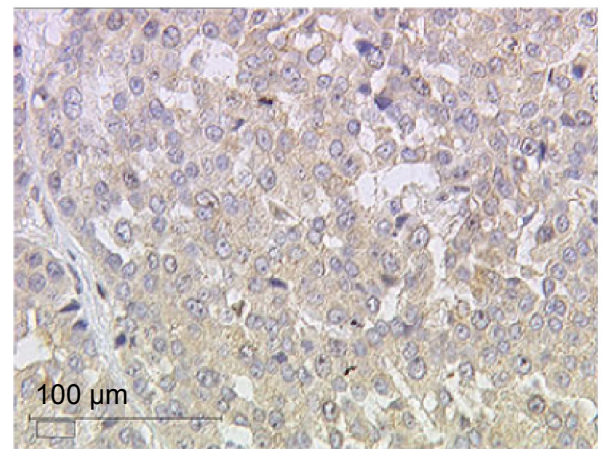

FOXP3

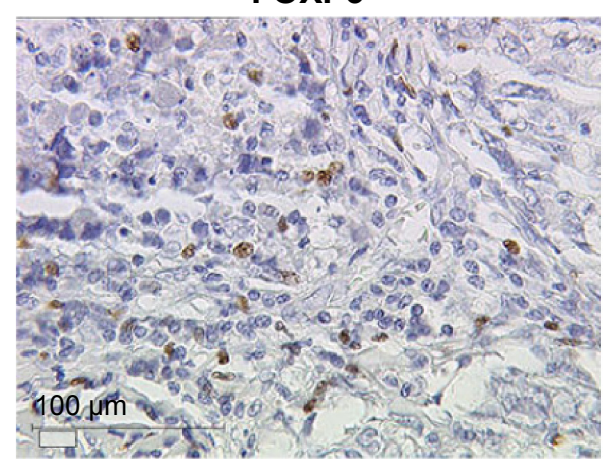

MCL1

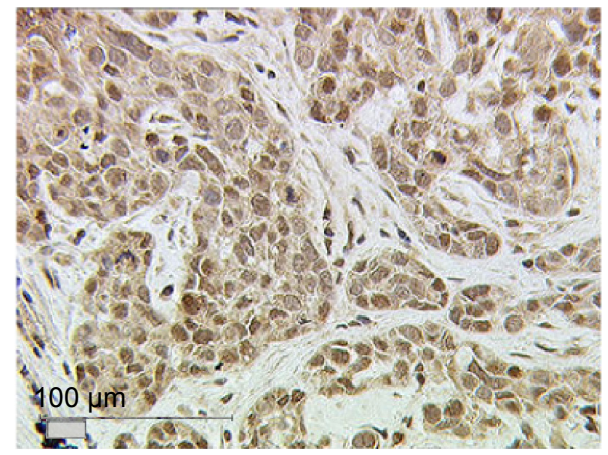

LRP6

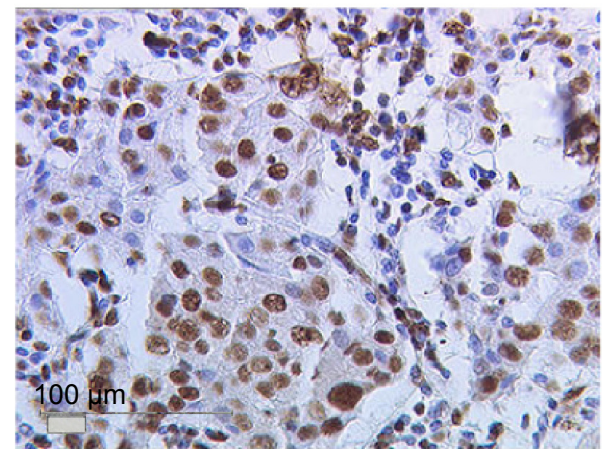

XBP1

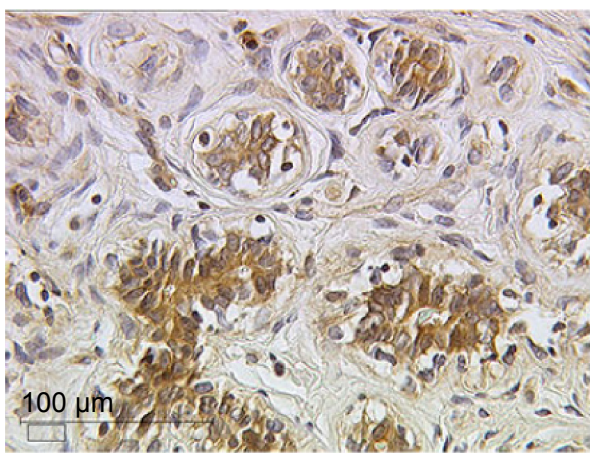

Figure I Staining of triple-negative breast cancer tissue with antibodies against molecules of different signal transduction cascades.

Notes: Blue: nuclear counterstain by hemalaun; brown: ABC staining of the respective signal transduction molecule. Magnification is 25 times.

Abbreviation: $\mathrm{ABC}$, Avidin-Biotin Complex.

localization of this molecule, a finding which has not yet been published. $\beta$-Catenin is an important signaling molecule in the development of TNBC, but no data on its role in patient survival were published so far. ${ }^{24}$ This finding was confirmed by our results. MCL1 in contrast is shown to be involved in enhanced metastasis formation and decreased DFS of patients with TNBC, and also for Notch1, a role in TNBC survival was shown recently. ${ }^{18} \mathrm{Cao}$ et al found 
that Notch1 is significantly correlated with lymph node status, a worse OAS, and DFS, and is an indicator of a poor prognosis. ${ }^{25}$ With the results we obtained from staining of a small number of TNBC tissue samples, we were not able to reassure these findings.

For LRP6 and XBP1, there are, at least up to now, no data available concerning their influence on survival of patients with TNBC. Some clues are found in the literature anyway, in order to elucidate the role of these two signal transduction molecules in survival of affected patients. LRP6 is known to stimulate tumor growth, and its inhibition was shown to suppress tumor growth in vivo, so it could be presumed that an overexpression of LRP6 has a negative influence on OAS, but on the other hand, it had been shown recently that LRP6 expression is inversely correlated with metastasis in mouse and human breast cancer, concluding that LRP6 could be used as a therapeutical target to prevent metastasis formation. ${ }^{26,27}$ The data we gained from our experiments
A

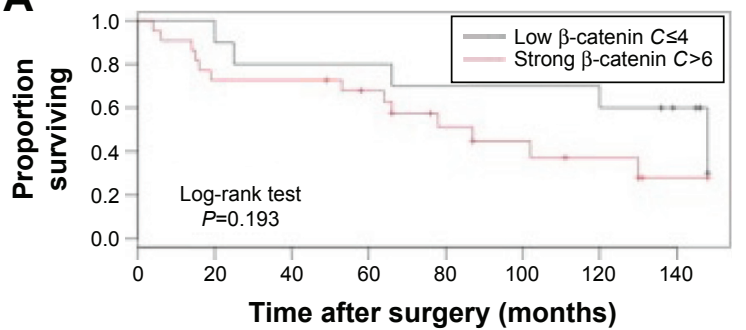

C

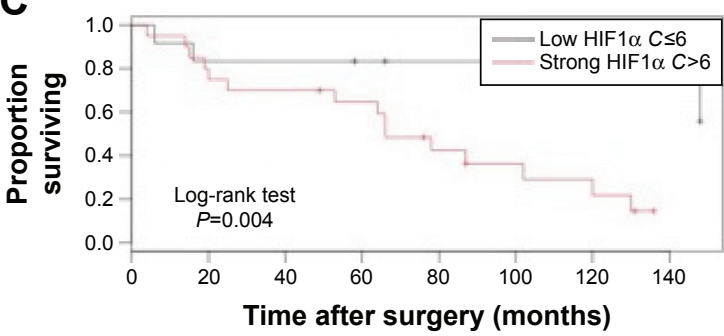

E

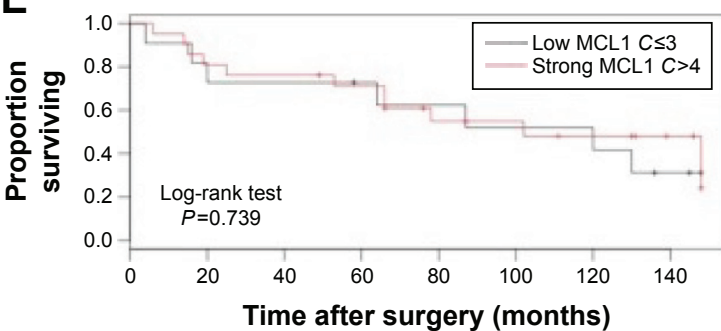

G
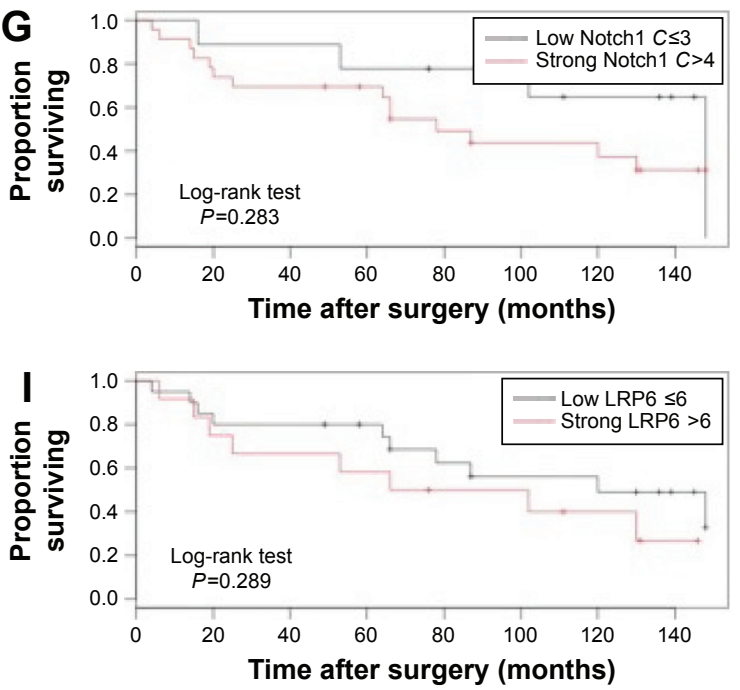

B

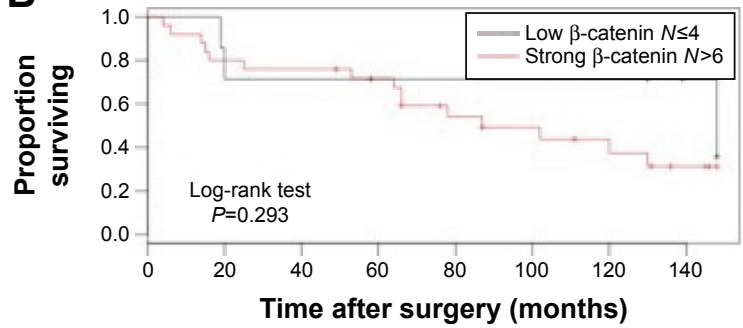

D

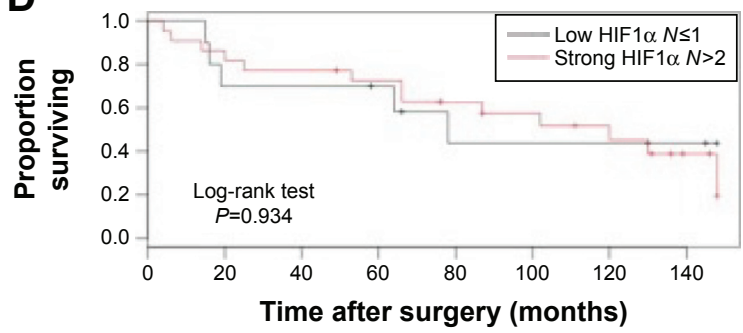

$\mathbf{F}$
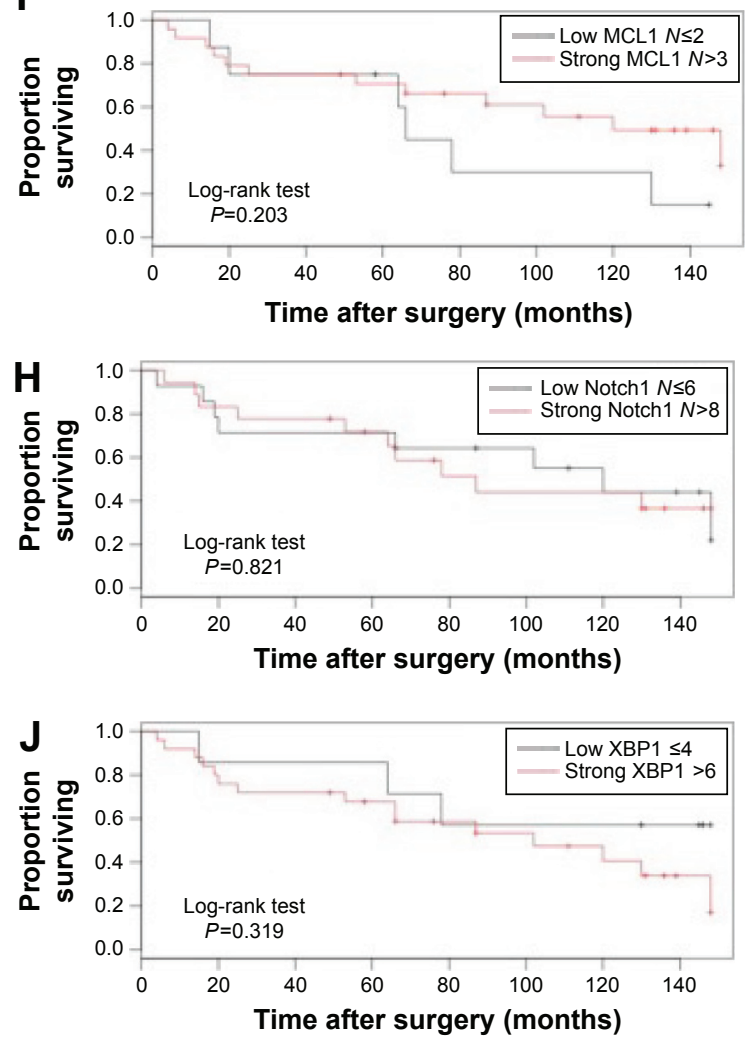

Figure 2 (Continued) 

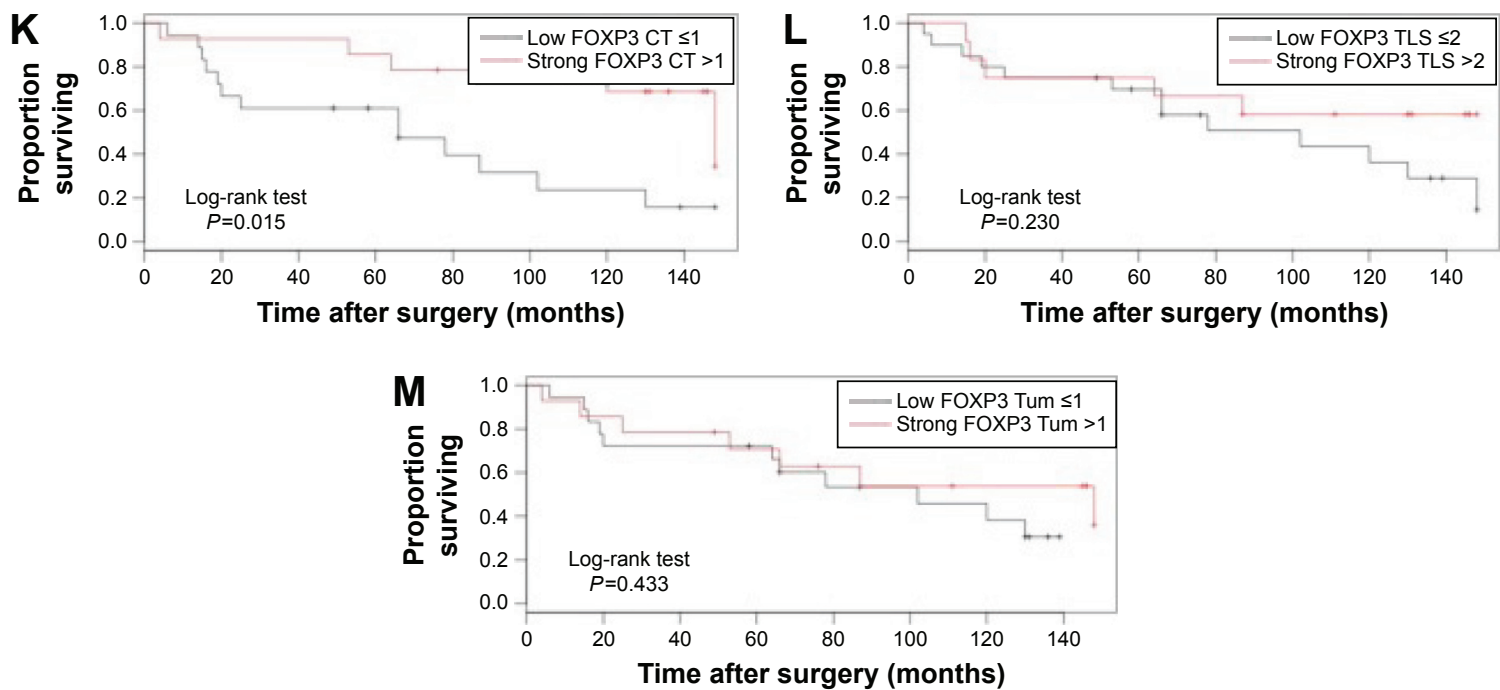

Figure 2 Kaplan-Meier survival curves in correlation to staining intensity.

Notes: Weak staining of the respective signal transduction molecule was compared to strong staining in respect to overall survival. If a staining was regarded as low or strong was determined with the median of Immune Reactive Score values. CT stands for centro-tumoral, meaning that lymphocytes in the center of the tumor are stained; Tum stands for a staining of tumor cells themselves. The $P$-value calculated from the log-rank test shows the statistical relevance. The survival curves of low and strong staining are different if $P \leq 0.05$

Abbreviations: $\mathrm{N}$, nuclear staining; $\mathrm{C}$, cytoplasmic staining; TLS, tumor-infiltrating lymphocytes.

support the latter finding. A certain function of XBP1 in TNBC survival was shown in coherence with the inducible nitric oxide synthase pathway, as inducible nitric oxide synthase inhibitors impaired, among others, endoplasmatic stress pathways, represented by IRE1 $\alpha$ and XBP1. This impairment significantly reduced tumor growth and metastasis formation. ${ }^{28}$ However, no direct correlation between XBP1 and OAS is described until now, and our data also indicate that there is no coherence.

Again, in accordance with our results are the data published for FOXP3 as a marker for poor prognosis in breast cancer survival. ${ }^{29}$ More in detail, no prognostic significance was found for FOXP3 expression in tumor cells and patient

Table 3 Statistical correlation of survival and staining intensity

\begin{tabular}{ll}
\hline Signal molecule stained & P-value \\
\hline$\beta$-Catenin - cytoplasmic staining & 0.193 \\
$\beta$-Aatenin - nuclear staining & 0.293 \\
HIFI $\alpha$ - cytoplasmic staining & $0.004 * *$ \\
HIFI $\alpha$ - nuclear staining & 0.934 \\
LRP6 & 0.289 \\
MCLI - cytoplasmic staining & 0.739 \\
MCLI - nuclear staining & 0.203 \\
Notch I - cytoplasmic staining & 0.283 \\
Notch I - nuclear staining & 0.821 \\
XBPI & 0.319 \\
FOXP3 - CT & $0.015^{*}$ \\
FOXP3 - TLS & 0.230 \\
FOXP3 - Tum & 0.433
\end{tabular}

Notes: *Significant at $P<0.05$ and **Significant at $P<0.005$.

Abbreviations: CT, centro-tumoral lymphocytes, TLS, tumor-infiltrating lymphocytes; Tum, tumor cells. survival, but FOXP3-positive lymphocytes were significantly associated with a poor OAS.

As a conclusion, it can be stated that signal transduction pathways are the upcoming targets for a new therapeutical option in the treatment of TNBC, but still lots of research is necessary to clarify the role of such signal transduction molecules and to find out which are the most suitable for cancer treatment. In our special case, it would mean to apply the technique of immunohistochemical staining of tissue samples with subsequent retrospective analysis of patient follow-up to a higher number of samples, to increase statistical significance and thereby to reinforce our findings.

\section{Acknowledgment}

The authors would like to thank Christoph Freier for help with statistical analysis.

\section{Disclosure}

The authors report no conflicts of interest in this work.

\section{References}

1. WHO. 2015 [cited 2015]. Available from: www.who.int/cancer/ detection/breastcancer/en/. Accessed November 17, 2015.

2. www.cancer.gov [webpage on the internet]. 2015 [cited 2015]. Available from: http://seer.cancer.gov/statfacts/html/breast.html. Accesssed November 17, 2015.

3. Badve S, Dabbs DJ, Schnitt SJ, et al. Basal-like and triple-negative breast cancers: a critical review with an emphasis on the implications for pathologists and oncologists. Mod Pathol. 2011;24(2):157-167.

4. Brenton JD, Carey LA, Ahmed AA, Caldas C. Molecular classification and molecular forecasting of breast cancer: ready for clinical application? J Clin Oncol. 2005;23(29):7350-7360. 
5. Kennecke H, Yerushalmi R, Woods R, et al. Metastatic behavior of breast cancer subtypes. J Clin Oncol. 2010;28(20):3271-3277.

6. Hugh J, Hanson J, Cheang MC, et al. Breast cancer subtypes and response to docetaxel in node-positive breast cancer: use of an immunohistochemical definition in the BCIRG 001 trial. JClin Oncol. 2009; 27(8):1168-1176.

7. Mehta RS. Dose-dense and/or metronomic schedules of specific chemotherapies consolidate the chemosensitivity of triple-negative breast cancer: a step toward reversing triple-negative paradox. J Clin Oncol. 2008;26(19):3286-3288; author reply 8.

8. Linderholm BK, Hellborg H, Johansson U, et al. Significantly higher levels of vascular endothelial growth factor (VEGF) and shorter survival times for patients with primary operable triple-negative breast cancer. Ann Oncol. 2009;20(10):1639-1646.

9. O'Shaughnessy J, Osborne C, Pippen JE, et al. Iniparib plus chemotherapy in metastatic triple-negative breast cancer. N Engl J Med. 2011; 364(3):205-214.

10. Schutz FA, Jardim DL, Je Y, Choueiri TK. Haematologic toxicities associated with the addition of bevacizumab in cancer patients. Eur J Cancer. 2011;47(8):1161-1174.

11. von Minckwitz G, Eidtmann H, Rezai M, et al. Neoadjuvant chemotherapy and bevacizumab for HER2-negative breast cancer. $N$ Engl $J$ Med. 2012;366(4):299-309.

12. Qiu M, Peng Q, Jiang I, et al. Specific inhibition of Notch1 signaling enhances the antitumor efficacy of chemotherapy in triple negative breast cancer through reduction of cancer stem cells. Cancer Lett. 2013;328(2):261-270.

13. Zhu H, Bhaijee F, Ishaq N, et al. Correlation of Notch1, pAKT and nuclear NF-kappaB expression in triple negative breast cancer. $\mathrm{Am}$ J Cancer Res. 2013;3(2):230-239.

14. Schwab LP, Peacock DL, Majumdar D, et al. Hypoxia-inducible factor 1alpha promotes primary tumor growth and tumor-initiating cell activity in breast cancer. Breast Cancer Res. 2012;14(1):R6.

15. Chen X, Iliopoulos D, Zhang Q, et al. XBP1 promotes triple-negative breast cancer by controlling the HIF1alpha pathway. Nature. 2014; 508(7494):103-107.

16. Kim MH, Koo JS, Lee S. FOXP3 expression is related to high Ki-67 index and poor prognosis in lymph node-positive breast cancer patients. Oncology. 2013;85(2):128-136.
17. Mahamodhossen YA, Liu W, Rong-Rong Z. Triple-negative breast cancer: new perspectives for novel therapies. Med Oncol. 2013;30(3): 653.

18. Yang L, Perez AA, Fujie S, et al. Wnt modulates MCL1 to control cell survival in triple negative breast cancer. BMC Cancer. 2014;14:124.

19. Lopes LF, Guembarovski RL, Guembarovski AL, et al. FOXP3 transcription factor: a candidate marker for susceptibility and prognosis in triple negative breast cancer. Biomed Res Int. 2014;2014:341654.

20. Takenaka M, Seki N, Toh U, et al. FOXP3 expression in tumor cells and tumor-infiltrating lymphocytes is associated with breast cancer prognosis. Mol Clin Oncol. 2013;1(4):625-632.

21. Remmele W, Stegner HE. [Recommendation for uniform definition of an immunoreactive score (IRS) for immunohistochemical estrogen receptor detection (ER-ICA) in breast cancer tissue]. Pathologe 1987;8(3):138-140. German.

22. Kaya AO, Gunel N, Benekli M, et al. Hypoxia inducible factor-1 alpha and carbonic anhydrase IX overexpression are associated with poor survival in breast cancer patients. J BUON. 2012;17(4):663-668.

23. Rajkovic-Molek K, Mustac E, Hadzisejdic I, Jonjic N. The prognostic importance of nuclear factor kappaB and hypoxia-inducible factor 1alpha in relation to the breast cancer subtype and the overall survival. Appl Immunohistochem Mol Morphol. 2014;22(6):464-470.

24. Xu J, Prosperi JR, Choudhury N, Olopade OI, Goss KH. Beta-Catenin is required for the tumorigenic behavior of triple-negative breast cancer cells. PLoS One. 2015;10(2):e0117097.

25. Cao YW, Li WQ, Wan GX, et al. Correlation and prognostic value of SIRT1 and Notch1 signaling in breast cancer. $J$ Exp Clin Cancer Res. 2014;33:97.

26. King TD, Suto MJ, Li Y. The Wnt/beta-catenin signaling pathway: a potential therapeutic target in the treatment of triple negative breast cancer. J Cell Biochem. 2012;113(1):13-18.

27. Ren DN, Chen J, Li Z, et al. LRP5/6 directly bind to Frizzled and prevent Frizzled-regulated tumour metastasis. Nat Commun. 2015;6:6906.

28. Granados-Principal S, Liu Y, Guevara ML, et al. Inhibition of iNOS as a novel effective targeted therapy against triple-negative breast cancer. Breast Cancer Res. 2015;17:25.

29. Kim W, Kim SY, Kim T, et al. ADP-ribosylation factors 1 and 6 regulate Wnt/beta-catenin signaling via control of LRP6 phosphorylation. Oncogene. 2013;32(28):3390-3396.
OncoTargets and Therapy

\section{Publish your work in this journal}

OncoTargets and Therapy is an international, peer-reviewed, open access journal focusing on the pathological basis of all cancers, potential targets for therapy and treatment protocols employed to improve the management of cancer patients. The journal also focuses on the impact of management programs and new therapeutic agents and protocols on

\section{Dovepress}

patient perspectives such as quality of life, adherence and satisfaction. The manuscript management system is completely online and includes a very quick and fair peer-review system, which is all easy to use. Visit http://www.dovepress.com/testimonials.php to read real quotes from published authors. 